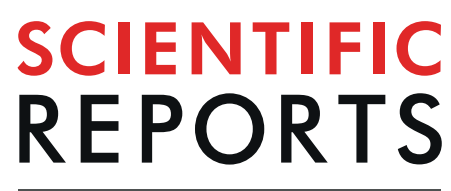

natureresearch

Check for updates

\title{
Assessment of haptoglobin alleles in autism spectrum disorders
}

Francesca Anna Cupaioli $\mathbb{C}^{1}$, Ettore Mosca $\mathbb{1}^{1}{ }^{1}$, Chiara Magri $\mathbb{C}^{2}$, Massimo Gennarelli ${ }^{2,3}$, Marco Moscatelli ${ }^{1}$, Maria Elisabetta Raggi ${ }^{4}$, Martina Landini ${ }^{1}$, Nadia Galluccio $\mathbb{D}^{1}$, Laura Villa ${ }^{4}$, Arianna Bonfanti ${ }^{4}$, Alessandra Renieri ${ }^{5,6}$, Chiara Fallerini ${ }^{5}$, Alessandra Minelli ${ }^{2}$, Anna Marabotti $\circledast^{7}$, Luciano Milanesi $\mathbb{1}^{1}$, Alessio Fasano $\mathbb{D}^{8,9}$ \& Alessandra Mezzelani $\mathbb{D}^{1 \bowtie}$

Gene-environment interactions, by means of abnormal macromolecular intestinal adsorption, is one of the possible causes of autism spectrum disorders (ASD) predominantly in patients with gastrointestinal disorders. Pre-haptoglobin-2 (zonulin), encoded by the Haptoglobin (HP) allele-2 gene, enhances the intestinal permeability by modulation of intercellular tight junctions. The two alleles of $H P, H P 1$ and $H P 2$, differ for 2 extra exons in HP2 that result in exon duplication undetectable by classic genomewide association studies. To evaluate the role of HP2 in ASD pathogenesis and to set up a method to discriminate HP alleles, Italian subjects with ASD $(n=398)$ and healthy controls $(n=379)$ were genotyped by PCR analysis; subsequently, the PCR results were integrated with microarray genotypes (Illumina Human Omni 1S-8), obtained using a subset from the same subjects, and then we developed a computational method to predict $H P$ alleles. On the contrary to our expectations, there was no association between $H P 2$ and ASD $(P>0.05)$, and there was no significant allele association in subjects with ASD with or without gastrointestinal disorders $(P>0.05)$. With the aid of bioinformatics analysis, from a window frame of $\sim 2 \mathrm{Mb}$ containing 314 SNPs, we obtain imputation accuracy $\left(r^{2}\right)$ between 0.4 and 0.9 (median 0.7 ) and correct predictions were between $70 \%$ and $100 \%$ (median $90 \%$ ). The conclusions endorse that enhanced intestinal permeability in subjects with ASD should not be imputed to HP2 but to other members of the zonulin family and/or to environmental factors.

Autism spectrum disorders (ASD) are a group of neurodevelopmental disorders with a complex and heterogeneous etiology that appears within the 3rd year of subjects and affects mainly males (ratio at 8 years of age male:female 4,5:1) ${ }^{1}$. Subjects with ASD are characterized by impaired interpersonal relationship, verbal and non-verbal communication difficulty, restricted and repetitive behavior.

ASD are often associated with comorbidities such as mental retardation, epilepsy, metabolic impairment, autoimmune and gastrointestinal disorders (GIDs) with microbial dysbiosis and enhanced intestinal permeability ${ }^{2-5}$.

Over the last decades, a dramatic increase of the disorder has occurred. This is justifiable to a limited extent, due to changes in diagnostic criteria in the Diagnostic and Statistical Manual of Mental Disorders (DSM) over the years. Genetics play a key role in autism pathogenesis and hundreds of genes have been associated to ASD, only a mere $20-25 \%$ of autistic subjects carry a causative genetic variant ${ }^{6}$. A gene-environment interaction has been proposed for the remaining cases, especially in cases with GIDs that correlate with the severity of ASD symptoms $s^{7,8}$. GIDs cases are often associated to "leaky gut" and the alteration in normal commensal gut microbiota (dysbiosis) ${ }^{7}$ leading to an increase in inflammation, abnormal macromolecule trafficking, potential translocation of intestinal microorganisms to lamina propria and blood ${ }^{9}$. Furthermore, dysbiosis is coupled with the production of toxins and an adsorption dysfunction. Many environmental factors including intestinal microorganisms (i.e. Candida

\footnotetext{
${ }^{1}$ Institute of Biomedical Technologies, National Research Council, Via Fratelli Cervi 93, 20090, Segrate, Italy. ${ }^{2}$ Department of Molecular and Translational Medicine, Biology and Genetic Unit, University of Brescia, 25123, Brescia, Italy. ${ }^{3}$ Genetics Unit, IRCCS Istituto Centro S. Giovanni di Dio, Fatebenefratelli, 25123, Brescia, Italy. ${ }^{4}$ Scientific Institute, IRCCS Eugenio Medea, Bosisio Parini, Lecco, Italy. ${ }^{5}$ Medical Genetics, University of Siena, Siena, Italy. ${ }^{6}$ Genetica Medica, Azienda Ospedaliera Universitaria Senese, Siena, Italy. ${ }^{7}$ Department Chemistry and Biology, "A. Zambelli", University of Salerno, Via Giovanni Paolo II 132, 84084, Fisciano, (SA), Italy. ${ }^{8}$ Mucosal Immunology and Biology Research Center, Center for Celiac Research and Treatment, and Division of Pediatric Gastroenterology and Nutrition, Massachusetts General Hospital - Harvard Medical School, 55 Fruit Street, Boston, MA, 02114, USA. ${ }^{9}$ European Biomedical Research Institute of Salerno (EBRIS), EBRIS Foundation, Via Salvatore de Renzi, 3, Salerno, 84125, Italy. ${ }^{凶}$ e-mail: alessandra.mezzelani@itb.cnr.it
} 
a

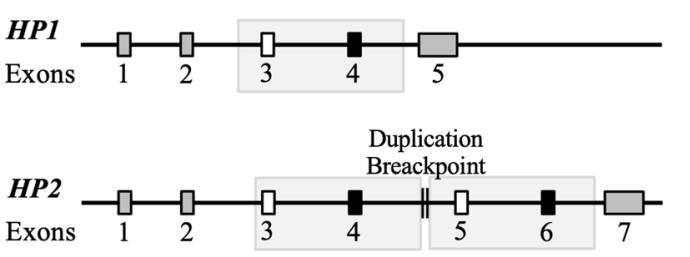

b

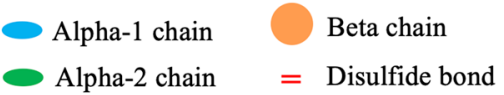

HP1

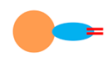

HP1-1
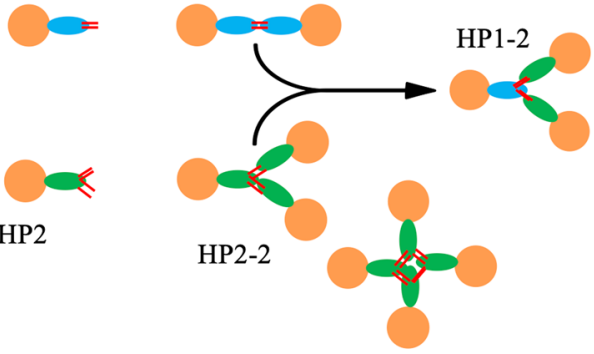

$347 / 406$

d 18 Signal peptide

Figure 1. $H P$ alleles and protein structures. (a) $H P 1$ and $H P 2$ allele structure; $H P 1$ allele contains 5 exons, while $H P 2$ is made up of 7 exons: exons 1 to 4 correspond to HP1, exons 5 and 6 is the duplication of exon 3 and 4, and exon 7 corresponds to exon 5 in HP1. (b) HP isoform and protein structure. HP1 allele encode for alpha-1 and beta chain (in blue and orange, respectively), while HP2 allele for alpha-2 and beta chain (green and orange respectively). The quaternary structures of $H P$ genotypes are also illustrated (modified from ${ }^{17}$ ). (c) $H P$ gene encode for signal peptide, from amino-acid residues 1-18, and mature protein sequence from 19 to 347 or 406, depending on the allele of origin: $H P 1$ allele encoding for alpha- 1 chain (residues 19-101) and beta chain (d), HP2 allele for alpha-2 chain (residues 19-160) and beta chain (e). Alpha-1 chain lacks of residues 29-87 of alpha-2 chain, and beta chain is common to HP1 and HP2 alleles.

albicans), bacterial toxins such as zonula occludens toxin (Zot) secreted by the pathogen Vibrio cholerae as well as food mycotoxins can alter the intestinal permeability ${ }^{10}$. Zot is a well-known toxin that induces severe intestinal permeability, that leads to the reversible opening of intestinal intercellular tight junctions via dissociation of Zonula Occludens-1 from its transmembrane binding partners occludin and claudin- $1^{11}$. A human protein called zonulin has been identified and resembles similarities with Zot. The human zonulin, that corresponds to pre-haptoglobin 2 (pre-HP2), shares a common motif with the active fragment of Zot that is critical for intestinal receptor binding. Pre-HP2 increases intestinal permeability in ex vivo and in vivo murine intestine in a dose-dependent manner, but not mature HP2 ${ }^{12}$. For these reasons, the role of human pre-HP2 in modulation of intestinal permeability is suggested due to its similarity with $\mathrm{Zot}^{10,13}$ in subjects with HP2 allele.

Mature HP, is an acute phase protein with a haemoglobin-binding function. Its presence is abundant in plasma where it acts as a free haemoglobin scavenger preventing oxidative damage between heme iron and protein or lipids. It also has an important role as an angiogenic, anti-inflammatory and immune modulator factor ${ }^{14}$. HP is mainly expressed in the liver and its synthesis is induced by cytokines, especially interleukin (IL) -6, IL-1 and tumour necrosis factor $(\mathrm{TNF})^{15}$.

In humans, HP (UniProt accession number P00738; GI: 386783) is encoded by 2 alleles, HP1 and HP2 (Gene ID: 3240) ${ }^{16}$ yielding three different genotypes: HP1-1, HP1-2 and HP2-2. HP1 and HP2 alleles differ for the duplication of exons 3 and 4, resulting in exons 5 and 6 in HP2 (Fig. 1). The precursor protein (pre-HP) includes signal peptide (amino-acids position: 1-18) and mature protein sequences, containing both alpha and beta chains. The proteolitically cleaved beta chain is common in HP from HP1 and HP2 alleles, while alpha chain differs from HP1 to HP2: the alpha chain from HP2 (alpha-2 chain) includes amino-acids at position 19-160, while alpha chain from HP1 (alpha-1 chain), lacks of residues 29-87 in comparison to alpha-2 chain, and includes residues from 19 to 101 (Fig. 1). The exon 3 contains the protein multimerization domain, allowing quaternary structure formation. Alpha and beta chains combine as tetramer to obtain HP. As a consequence of $H P$ allelic variant, the structure of HP in HP1-1 phenotype is (alpha-1-beta) $)_{2}$, that of HP1-2 are (alpha-1-beta) ${ }_{2}$ and (alpha-2-beta) ${ }_{n}$, and that of HP2-2 is (alpha-2-beta) ${ }_{n}^{17,18}$ (Fig. 1). HP1, on the other hand, displays fast (HP1F) and slow (HP1S) isoforms of the alpha chain, depend on their fast (F) or slow (S) electrophoretic motilities. These two forms differ for the presence of an amino-acid substitution (Lys54 of HP1S is replaced by Glu in the HP1F) ${ }^{19}$ and originate from different allelic subtypes: HP1F, HP1S, HP2FF, HP2FS and HP2SS (very rare). Because of the structure of 
the two alleles, it is very difficult to recognize them by means of classic genome-wide association studies (GWAS), so most of the genotyping studies are limited to small size populations analyzed by PCR or quantitative RT-PCR.

HP1 and HP2 proteins are functionally different. HP1-1 protein has a superior antioxidant capacity than HP2-2 and induces a more rapid free hemoglobin degradation ${ }^{17,20}, H P 1$ carriers also have a higher HP concentration than HP2, with HP1-1 > HP1-2 > HP2-2 21 .

Due to the protective role of HP1, HP1-1 genotype has a significant association to longevity ${ }^{22}$, on the other hand, HP2-2 has a higher susceptibility to immune-mediated diseases, such as celiac disease, Rheumatoid Arthritis, Type 1 diabetes, Systemic Lupus Erythematosus ${ }^{23-26}$ and cancer.

In 2000, Wang and collaborators reported that an 8 amino-acid sequence in N-terminal of human pre-HP2 (residues 8-15: G G V L V Q P G) shared a common motif with the active fragment of Zot (residues 291-298-: G R L C V Q D G) from which originated the alternative name "zonulin". The common amino-acid motif is: non-polar $(G)$, variable, non-polar, variable, non-polar (V), polar (Q), variable, non-polar $(G)^{13}$. In 2018, Scheffler and collaborators identified another member of the zonulin family, properdin, a mannose-associated serine protease $\mathrm{e}^{27}$.

Zonulin induces the disassembling of intercellular tight junctions in many epithelial and endothelial barriers, including the "leaky gut" and enhancing the intestinal trafficking of microorganisms and macromolecules that, in sequence, challenge the immune response involving different tissues and organs in genetically susceptible subjects ${ }^{12,26,28}$.

Of interest, a significant high level of zonulin (pre-HP2) in a small cohort of subjects with ASD vs healthy controls was found ${ }^{29}$, as well as an association between HP2 allele and autism with GID comorbidity ${ }^{30}$.

In this study, we genotyped a larger cohort of patients and controls to test the hypothesis that HP2 allele is associated with ASD development at least with GID in autism. We assessed the $H P$ alleles in a batch of Italian subjects with ASD $(n=398)$ and healthy controls $(n=380)$ by PCR analysis. Because of the internal exon duplication of $H P$, it is very difficult to distinguish the two different alleles by means of standard bioinformatics analysis, and PCR techniques limit the size of tested subjects. We integrated PCR and microarray genotyping data, previously obtained on a subgroup of patients and controls, to impute and discriminate $H P$ alleles by GWAS. Thus, we provide a bioinformatic reference useful for further $H P$ prediction on publicly available GWAS data.

\section{Materials and Methods Ethics committees.}

- Ethics committee IRCCS Eugenio Medea - Scientific section of the association "La Nostra Famiglia” 2010, IRCCS Eugenio Medea - La Nostra Famiglia, Bosisio Parini (LC), Italy;

- Ethics committee of the hospital institutions (CEIOC) IRCCS Istituto Centro San Giovanni di Dio Fatebenefratelli 50/2008, IRCCS Istituto Centro S. Giovanni di Dio Fatebenefratelli, Brescia, Italy;

- Local ethics committee, Prot. 567/06 dated 28-09-2006 and document integration dated 09-10-2006, Azienda Ospedaliera Universitaria Senese, Siena, Italy.

All Ethics Committees of involved Institutions approved the study and enrolled subjects that signed informed consents. Informed signed consents were collected from parents and/or legal guardians for subjects under the age of 18 years.

All methods were performed in accordance with relevant guidelines and regulations regarding observational studies.

Subjects. A total sample-set consisting of 398 Italian subjects with ASD [338 males (85\%) and 60 females (15\%)], and 379 unrelated Italian controls [170 males (45\%) and 209 females (55\%)] were recruited and peripheral blood samples collected.

Patients. IRCCS Eugenio Medea - La Nostra Famiglia recruited 206 subjects with ASD [171 males (83\%) and 35 females (14\%)] ranging between 2 and 12 years old. The diagnosis for autism (70.3\%), Asperger's syndrome (3.5\%) or pervasive developmental disorder not otherwise specified (26.2\%) were performed according to DSM, fourth edition, text revision (DSM-IV TR) (American Psychiatric Association [APA], 2000) and by the Autism Diagnostic Observation Scale 2 (ADOS-2) ${ }^{31}$ and Autism Diagnostic Interview - Revised (ADI-R) ${ }^{32}$. Subjects with genetic syndromes, epilepsy and neuroradiological confirmed disorders were excluded. Clinical data about the presence/absence of GIDs were evaluated in a sub-group of 157 subjects with ASD [135 males (86\%) and 22 females (14\%)].

The University of Siena recruited 192 subjects with ASD [167 males (87\%) and 25 females (13\%)] ranging from 1 to 3 years old. The diagnosis for autism (82\%), Asperger's syndrome $(0,5 \%)$ and pervasive developmental disorders not otherwise specified (17\%) were performed according to the DSM-IV TR (APA, 2000), and by the ADOS-2 and ADI-R. Within these patients and clinical data about the presence/absence of GIDs were evaluated in a sub-group of 8 male subjects.

Controls. IRCCS Eugenio Medea - La Nostra Famiglia recruited 25 unrelated children (12 males and 13 females) evaluated by Child Behavior Checklist as neurotypical controls; the University of Brescia and IRCCS Istituto Centro S. Giovanni di Dio, Fatebenefratelli of Brescia enrolled 259 healthy subjects [109 males (42\%) and 150 females (58\%); mean age 51.6 \pm 15.5 ]; 191 (73.7\%) were unrelated non-affected volunteers who were screened for DSM-IV Axis I disorders by expert psychologists using the Mini-International Neuropsychiatric Interview ${ }^{33}$. Only subjects without a history of drug or alcohol abuse/dependence and without a personal or first-degree family history of psychiatric disorders were enrolled in this study. Furthermore, subjects who obtained a score lower than 27/30 at the Mini Mental State Examination ${ }^{34}$ were excluded. The University of Siena recruited 95 healthy 


\begin{tabular}{|c|c|c|c|}
\hline \multirow[b]{2}{*}{ Primer } & \multirow[b]{2}{*}{ Primer sequence } & \multicolumn{2}{|c|}{ Amplicon length } \\
\hline & & HP-1 & HP-2 \\
\hline NewA & 5'- GGGGTTCCTGCCAGAAATGA - $3^{\prime}$ & $1775 \mathrm{bp}$ & $3487 \mathrm{bp}$ \\
\hline NewB & 5'- CCCTGGCTGGTGAACTGTATT - $3^{\prime}$ & & \\
\hline NewC & 5'- ATGCCAACCTGCCTCGTATT - -3' & - & $360 \mathrm{bp}$ \\
\hline NewD & 5'- CGAACCGAGTGCTCCACATA -3' & & \\
\hline
\end{tabular}

Table 1. PCR primers sequence and amplicon length.

controls [ 45 females (47\%) and 50 males (53\%), aged between 20 years and 61 years, mean age of 41 years]. The subjects were healthy adults who were not evaluated for neuropsychiatric conditions.

DNA isolation. Genomic DNA was extracted from peripheral blood leukocytes, using commercial kits and relative protocols. Quality (280/260 ratio) and quantity of DNA was checked by NanoDrop 2000 spectrophotometer (Thermo Fisher Scientific, Wilmington, DE USA).

Haptoglobin genotyping. PCR - HP genotypes were determined by PCR amplification devised by Koch and collaborators ${ }^{35}$ with minor modifications. Briefly, new PCR primers (Table 1) were designed on HP1 and HP2 specific sequences M69197.1 (EMBL-GeneBank Data Libraries) and 30 ng of genomic DNA was amplified in $10 \mu \mathrm{l}$ of reaction mixture (Platinum ${ }^{\mathrm{TM}}$ SuperFi $^{\mathrm{TM}}$ PCR Master Mix, for primers NewA and NewB, and Platinum ${ }^{\circledR}$ PCR SuperMix, High Fidelity, for primers NewC and NewD- Invitrogen ${ }^{\mathrm{TM}}$, Thermo Fisher Scientific, Wilmington, DE USA) as suggested by the supplier. Two couples of primers, NewA with NewB and NewC with NewD were used separately, to analyze every sample and each analysis was duplicated. Primers NewA (5'- GGGGTTCCTGCCAGAAATGA - $3^{\prime}$ ) and NewB (5'- CCCTGGCTGGTGAACTGTATT - $\left.3^{\prime}\right)$ can produce, contextually, a 1775 bp and/or a 3487 bp specific product for HP1 and HP2 alleles, respectively. Primers NewC $\left(5^{\prime}\right.$ - ATGCCAACCTGCCTCGTATT - $\left.3^{\prime}\right)$ and NewD (5'- CGAACCGAGTGCTCCACATA - $\left.3^{\prime}\right)$ amplify a $360 \mathrm{bp}$ HP2 allele specific fragment.

After denaturation for 30 seconds at $98^{\circ} \mathrm{C}$, thermo-cycling profile consisted of denaturation for 12 seconds at $98^{\circ} \mathrm{C}$, annealing for 10 seconds at $65.4^{\circ} \mathrm{C}$ and extension for 1 minute and 40 seconds at $72^{\circ} \mathrm{C}$ repeated for 35 cycles when using primers NewA and NewB, while denaturation for 2 minutes at $94^{\circ} \mathrm{C}$, thermo-cycling profile for 1 minutes at $94^{\circ} \mathrm{C}, 40$ seconds at $68^{\circ} \mathrm{C}$ and 50 seconds at $72^{\circ} \mathrm{C}$ repeated for 32 cycles, and extension for 2 minutes at $72^{\circ} \mathrm{C}$ for primers NewC and NewD. PCR products obtained by NewA-B and NewC-D couples of primers were run on $1 \%$ and $2 \%$ agarose gel (E-Gel agarose gels, Invitrogen ${ }^{\mathrm{TM}}$, Thermo Fisher Scientific) respectively. In HP1-1 we detected the NewA-B PCR product of 1775 bp length, but not NewC-D PCR product; in HP2-2 we found the NewA-B PCR product of $3487 \mathrm{bp}$ and NewC-D $360 \mathrm{bp} \mathrm{PCR} \mathrm{product;} \mathrm{in} \mathrm{HP1-2} \mathrm{subjects} \mathrm{we} \mathrm{identified} \mathrm{both} 3487 \mathrm{bp}$ and $1775 \mathrm{bp}$ amplicons, and the $360 \mathrm{bp}$ PCR product (Fig. 2).

Sanger sequencing. To check the PCR products, two PCR products for each type of $H P$ genotype obtained by NewA and -B amplification (HP1-1, HP1-2, HP2-2) as well as those obtained by NewC and -D, were purified (CleanSweep PCR Purification Reagent, Applied Biosystems, Thermo Fisher Scientific, Wilmington, DE USA) and sequenced by Sanger method using NewC and NewD respectively for forward and reverse sequencing. Results were compared to HP1 and HP2 sequences (NCBI Reference Sequence: NG_012651.1).

Data analysis. The association between $H P$ alleles and ASD was calculated considering subjects with ASD $v s$ all the analyzed controls and subjects with ASD without GID vs subjects with ASD with GID. Chi-square with Yates correction test was applied to evaluate statistical significance. We sub-classified the controls in two groups referring to the enrollment screening test: ASDs negative controls $(n=188)$, referred as non-ASD (NASD) controls, and those negative for ASD and DSM-IV Axis I disorders $(\mathrm{n}=191)$, referred as super-controls. The HP allelic distribution was evaluated in all subjects with ASD and controls and in all sub-groups. Possible effect of sex on HP allele distribution was investigated in patients with ASD and in healthy subjects, considering the sub-classification of controls.

Due to the small size of controls enrolled in this study we implemented our data by a meta-analysis including $H P$ genotyping of healthy Italians ${ }^{22,36}$, healthy Caucasians ${ }^{35}$ (Table 2).

Microarray. Microarray analysis was performed on a subset of samples $(n=318)$ collected by IRCCS Eugenio Medea - La Nostra Famiglia using Human Omni 1S-8 v 1.0 Illumina chip and iScan Illumina (Illumina, San Diego, CA, USA) according to the manufacturer's protocols, and a selection of 230 subjects were genotyped by microarray and PCR.

Among controls collected by the University of Brescia, 177 subjects ( 87 females and 90 males) were genotyped by Affymetrix Human Mapping GeneChip 6.0 array (Affymetrix, Thermo Fisher Scientific, Wilmington, DE USA) according to the manufacturer's protocols and applying quality control procedures described by Sacchetti and colleagues ${ }^{37}$, and 103 of them were genotyped for HP gene by PCR.

Bioinformatics. Genetic data for 1,185,076 SNPs in 230 subjects were collected from "Top" alleles of the Illumina's Genome Studio Final Report, generated using HumanOmni1S platform and GRCh37.p13 genome assembly. Standard quality control procedures were used to exclude individuals with discordant sex and call rates 
$\mathbf{a}$

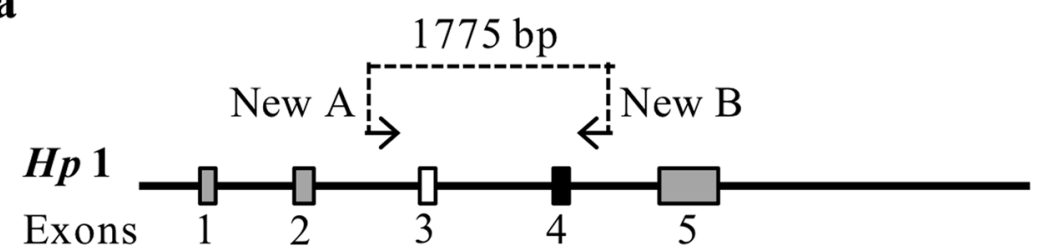

b

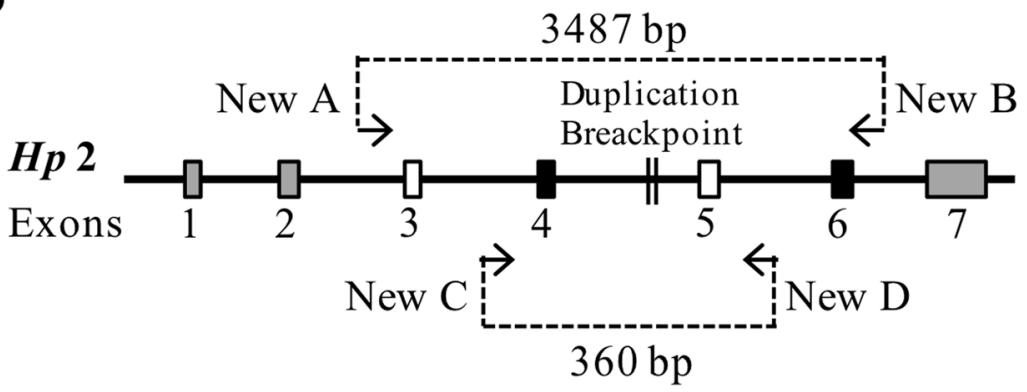

c

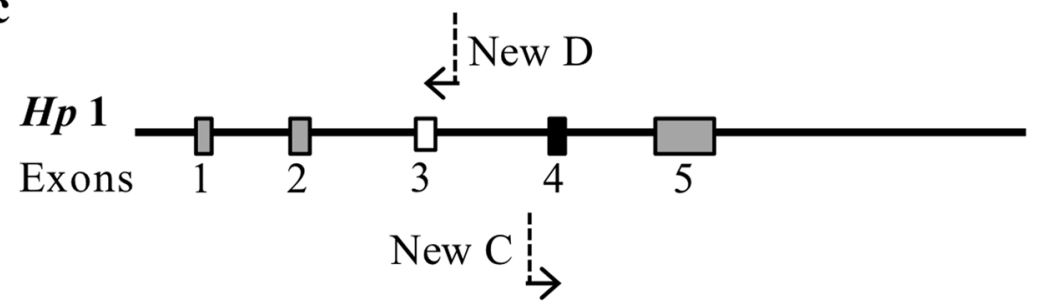

d

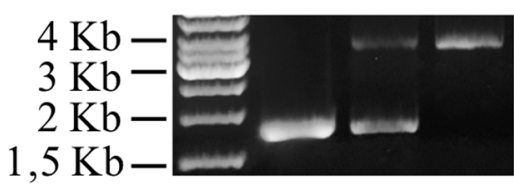

e

$1-1 \quad 1-2 \quad 2-2$

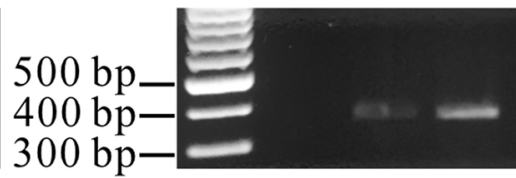

$1-1 \quad 1-2 \quad 2-2$

Figure 2. $H P$ alleles structure and PCR primers. HP2 allele contains a duplication of $1700 \mathrm{bp}$, corresponding to extra exons 3 and 4. Due to this duplication, PCR amplification with NewA and NewB primers (narrowly indicate $5^{\prime}>3^{\prime}$ orientation) amplification gives $1775 \mathrm{bp}$ length amplicons in HP1 allele (a), and $3487 \mathrm{bp}$ length amplicons in HP2 allele (b). PCR products obtained with primers pairs NewC/NewD are 360 bp long in HP2 allele (b), while in HP1 no amplicon is given (c). Agarose gels, $1 \%(\mathbf{d})$ and $2 \%(\mathbf{e})$, are used to detect the three different genotype for both primer pairs. In the case of NewA and NewB in HP1-1 subjects only 1775 bp amplicon is present, in HP1-2 both amplicons are present and in HP2-2 only $3487 \mathrm{bp}$ amplicon is detected (d). For NewC/NewD primer pairs $360 \mathrm{bp}$ amplicons is detected only in HP1-2 and HP2-2 genotypes (e). Gels images (d,e) are cropped from images of full-length gels (Supplementary Fig. S1).

below $98 \%$ and filter out SNPs with MAF $<1 \%$, Hardy Weinberg $p<1 \times 10^{-4}$ and a call rate $<99 \%$. A total of 696,849 markers and 293 subjects fulfill quality control requirements. The analysis of genetic similarity among individuals revealed the presence of several outliers. Only individuals belonging to the most populated cluster were considered for further analyses. The quality control procedures repeated on this subgroup determined the exclusion of some additional markers. A total of $690^{\prime} 215$ markers and 282 individuals were retained.

Genotypes detected by microarray and HP genotypes detected by PCR were phased using Beagle ${ }^{38}$ against the haplotypes provided by the 1000 Genomes project (Phase 3). HP alleles were imputed using Beagle v4.1 ${ }^{39}$. A total of 100 cross-validation trials were run, randomly assigning each time $90 \%$ of the subjects to the "reference" group and the remaining $10 \%$ to the "test" group.

\section{Results}

HP allele distribution in patients and controls. As for PCR HP genotyping the length of NewA-B PCR products reflected the expected lengths $(H P 1=1775 \mathrm{bp} ; H P 2=3487 \mathrm{bp})$ and included the duplication breakpoint sequence only in NewC-D products (360 bp) and in NewA-B products corresponding to HP2 allele (Fig. 2). 


\begin{tabular}{|c|c|c|c|c|c|c|c|c|c|c|c|}
\hline & & \multirow[b]{2}{*}{$\begin{array}{l}\text { Subjects with } \\
\text { ASD }\end{array}$} & \multicolumn{3}{|c|}{ Controls enrolled in this study } & \multicolumn{4}{|c|}{ Controls from literature } & \multirow[b]{2}{*}{$\begin{array}{l}\text { Subjects with } \\
\text { ASD without } \\
\text { GID }\end{array}$} & \multirow[b]{2}{*}{$\begin{array}{l}\text { Subjects with } \\
\text { ASD with } \\
\text { GID }\end{array}$} \\
\hline & & & Total & Super controls & NASD control & $\begin{array}{l}\text { Neurotypical } \\
\text { Italian } \\
\text { populations }\end{array}$ & \begin{tabular}{|l|} 
Total controls + \\
neurotypical Italian \\
population
\end{tabular} & $\begin{array}{l}\text { NASD controls }+ \\
\text { neurotypical Italian } \\
\text { populations }\end{array}$ & $\begin{array}{l}\text { Caucasian } \\
\text { healthy } \\
\text { subjects }\end{array}$ & & \\
\hline & & $\mathrm{n}=398$ & $\mathrm{n}=379$ & $n=191$ & $n=188$ & $n=2130$ & $n=2509$ & $n=2318$ & $\mathrm{n}=\mathbf{2 4 9}$ & $n=76$ & $n=89$ \\
\hline \multirow{3}{*}{ Genotype } & HP1-1 & $59(14.8 \%)$ & $35(9.2 \%)$ & $18(9.4 \%)$ & $17(9.1 \%)$ & $294(13.8 \%)$ & $329(13.1 \%)$ & $311(13.4 \%)$ & $36(14.5 \%)$ & $13(18.1 \%)$ & $12(14.1 \%)$ \\
\hline & HP1-2 & $175(44.0 \%)$ & $163(43.0 \%)$ & $68(35.6 \%)$ & $95(50.5 \%)$ & $913(42.9 \%)$ & $1076(42.9 \%)$ & $1008(43.5 \%)$ & $120(48.2 \%)$ & $25(34.7 \%)$ & $39(45.9 \%)$ \\
\hline & HP2-2 & $164(41.2 \%)$ & $181(47.8 \%)$ & $105(55.0 \%)$ & $76(40.4 \%)$ & $923(43.3 \%)$ & $1104(44.0 \%)$ & $999(43.1 \%)$ & $93(37.3 \%)$ & $34(47.2 \%)$ & $34(40.0 \%)$ \\
\hline \multirow{3}{*}{$\begin{array}{l}\text { Allele } \\
\text { frequency }\end{array}$} & HP1 & $293(36.8 \%)$ & $233(30.7 \%)$ & $104(27.2 \%)$ & $129(34.3 \%)$ & $1501(35.2 \%)$ & $1734(34.6 \%)$ & $1630(35.2 \%)$ & $192(38.6 \%)$ & $51(35.4 \%)$ & $63(37.1 \%)$ \\
\hline & HP2 & $503(63.2 \%)$ & $525(69.3 \%)$ & $278(72.3 \%)$ & $247(65.7 \%)$ & $2759(64.8 \%)$ & $3284(65.4 \%)$ & $3006(64.8 \%)$ & $306(61.4 \%)$ & $93(64.6 \%)$ & $107(62.9 \%)$ \\
\hline & P value & & $0.0134^{*}$ & $0.0014 * *$ & 0.4429 & \begin{tabular}{|l|}
0.4169 \\
\end{tabular} & 0.2480 & \begin{tabular}{|l|}
0.3904 \\
\end{tabular} & 0.5673 & & \begin{tabular}{|l|}
0.8542 \\
\end{tabular} \\
\hline
\end{tabular}

Table 2. HP allelic distribution in subjects with ASD and controls. Data on neurotypical Italian population were from the total of controls enrolled by Bottini and collaborators and Napolioni and co-worker ${ }^{22,36}$. Genotyping data on Caucasian healthy subjects were from Koch and collaborators ${ }^{35}$, and $H P$ allele frequency of individuals sampled by the 1000 Genomes Project was calculated in the study of Boettger and co-worker ${ }^{58}$. HP2 allele is highly represented in subjects with ASD and controls. HP1 allele increases significantly in patients when compared to total and non-ASDs controls enrolled in this study and to controls from 1000 Genomes projects. In contrast, $H P 1$ allele did not increase significantly when compared to the other controls. P value was calculated with Chi-square with Yates correction test $(* \mathrm{P}<0.05$; **P $<0.005)$.

HP genotypes of the 398 subjects with ASD were HP1-1=59 (14.8\%), HP1-2=175 (44.0\%) and HP2-2 = 164 (41.2\%), and the frequencies of HP1 and HP2 were 293 (36.8\%) and 525 (63.2\%), respectively. The distribution of genotype frequencies is in Hardy Weinberg equilibrium $\left(\mathrm{p} \approx 0.3 ; \chi^{2}\right.$ test $)$.

The genotypes of all the controls $(\mathrm{n}=379)$ were: HP1-1 = $35(9.2 \%), \mathrm{HP} 1-2=163(43.0 \%)$ and HP2-2 $=181$ $(47.8 \%) ; H P 1$ frequency $=233(30.7 \%)$ and $H P 2=525(69.3 \%)$; and are in Hardy Weinberg equilibrium $(\mathrm{p} \approx 0.9$; $\chi^{2}$ test). Considering the two control groups separately, the allelic distribution of $H P 1$ was $34,3 \%$ in NASD controls and decreased to $27,2 \%$ in super-controls, while HP2 was $65,7 \%$ and $72,3 \%$, respectively (Table 2 ).

The HP1 increased in subjects with ASD when compared to the total number of controls: Chi-square with Yates correction test was calculated and a significant increase of $H P 1$ allele frequency was found in subjects with ASD compared to total controls (NASD controls+super controls) $(\mathrm{P}<0.05)$ and super-controls $(\mathrm{P}<0,005)$. Consequently, the association between HP1 and ASD disappeared $(\mathrm{P}>0.05)$ excluding super-controls and adding to NASD controls, Italian and Caucasian healthy subjects ${ }^{22,35,36}$ (Table 2).

Considering only subjects with ASD with clinical GID data $(\mathrm{n}=157)$, the allelic distribution in subjects with ASD with GID ( $\mathrm{n}=89 ;$ HP1 37.1\% and HP2 62.9\%) and without GID ( $\mathrm{n}=76 ;$ HP1 35.4\% and HP2 64.6\%) did not differ $(\mathrm{P}>0.05)$ (Table 2$)$.

The sex ratio differs from the subjects with ASD and controls, and this could influence the $H P$ allelic distribution. For this reason, we also investigated $H P$ genotype related to sex. HP allelic distribution did not show statistically significant imbalanced sex ratio in patients with ASD $(\mathrm{P}>0.1)$, in the total number of controls $(\mathrm{P}>0.5)$, and in the sub-groups NASD controls and super controls $(\mathrm{P}>0.1)$ (Supplementary Table $\mathrm{S} 1$ ). To know more about the consequence of the sex imbalance within controls and subjects with ASD we also re-analyzed data from the study of Brackenridge $e^{40}$ that found a significant sex imbalance in HP1-2 distribution within Australian population. $H P$ allelic distribution was calculated for healthy Australian population (Supplementary Table S1) and we did not find significant difference in $H P$ distribution between males and females by Fisher test $(\mathrm{P}>0.5)$.

HP alleles imputation from SNP haplotypes. To assess whether $H P$ alleles can be predicted by the haplotype of surrounding SNPs we performed a series of cross-validation trials, in which we split the autistic population genotyped with the Illumina array into two groups ("reference" and "test") panels, and imputed (Beagle v4.1) the HP alleles of test subjects, using the multi-SNP haplotypes surrounding HP and HP alleles detected by PCR in subjects assigned to the reference group. We considered the SNPs occurring up to $2 \mathrm{MB}$ around $H P$ gene and found the highest accuracy for HP2-2 (0.97) using 79 SNPs (0.5 Mb window). Among HP genotypes, we found that genotype 1-1 was the most difficult to predict (Table 3 ).

The same approach was applied to healthy subjects, genotyped by the Affymetrix platform (Table 4). We observed a slightly lower accuracy (e.g. 0.89 using $76 \mathrm{SNPs}$; $0.5 \mathrm{Mb}$ window), but its trends relative to the number of surrounding SNPs considered and to HP genotypes were similar to those observed in autistic subjects.

\section{Discussion}

Wang and colleagues first found, in human intestinal tissue, a protein that they called zonulin, sharing a common amino-acid motif with Zot, the Vibrio cholera toxin acting on the intestinal wall disassembles tight junctions with consequent intestinal permeability ${ }^{13}$. A following study identified human zonulin, as pre-HP2 ${ }^{12}$. Since an involvement of zonulin has already been demonstrated in many immune mediated diseases with $\mathrm{GID}^{41}$, and many subjects with ASD suffer from GID and "leaky gut", a possible role of zonulin in ASD pathogenesis was assumed ${ }^{29}$. Nevertheless, because of its structure containing a duplication of 2 exons, HP gene is difficult to genotype by classic genome-wide studies and $H P$ available data are limited to a very small amount of cases.

To understand the role of zonulin in ASD etiology and to provide a bioinformatics method to genotype $H P$ using or re-using newly generated or archival data, respectively, we $i$ ) analyzed, by PCR, the HP allele distribution 


\begin{tabular}{|l|l|l|l|l|l|}
\hline \multirow{2}{*}{$\begin{array}{l}\text { Window } \\
(\mathbf{M b})\end{array}$} & \multirow{2}{*}{$\begin{array}{l}\text { Number of } \\
\text { markers }\end{array}$} & \multicolumn{4}{|l|}{ Accuracy of prediction } \\
\cline { 3 - 6 } & HP1-1 & HP1-2 & HP2-2 & Mean \\
\hline $\mathbf{0 , 1}$ & 16 & 0.34 & 0.63 & 0.96 & 0.73 \\
\hline $\mathbf{0 , 2 5}$ & 51 & 0.70 & 0.93 & 0.96 & 0.91 \\
\hline $\mathbf{0 , 5}$ & $\mathbf{7 9}$ & $\mathbf{0 . 8 0}$ & $\mathbf{0 . 9 2}$ & $\mathbf{0 . 9 7}$ & $\mathbf{0 . 9 2}$ \\
\hline $\mathbf{1}$ & 124 & 0.73 & 0.94 & 0.96 & 0.91 \\
\hline $\mathbf{1 , 5}$ & 203 & 0.76 & 0.93 & 0.98 & 0.92 \\
\hline $\mathbf{2}$ & 313 & 0.79 & 0.92 & 0.97 & 0.92 \\
\hline
\end{tabular}

Table 3. Bioinformatics imputation analysis, Illumina platform. Results of $H P$ alleles imputation from SNP haplotypes genotyped using an Illumina array. Best prediction accuracy results are achieved in increments of 0,5 windows (in bold).

\begin{tabular}{|l|l|l|l|l|l|}
\hline \multirow{2}{*}{$\begin{array}{l}\text { Window } \\
(\mathbf{M b})\end{array}$} & \multirow{2}{*}{$\begin{array}{l}\text { Number of } \\
\text { markers }\end{array}$} & \multicolumn{4}{|l}{ Accuracy of prediction } \\
\cline { 3 - 6 } & HP1-1 & HP1-2 & HP2-2 & Mean \\
\hline $\mathbf{0 , 1}$ & 11 & 0.26 & 0.38 & 0.94 & 0.67 \\
\hline $\mathbf{0 , 2 5}$ & 40 & 0.64 & 0.87 & 0.97 & 0.89 \\
\hline $\mathbf{0 , 5}$ & $\mathbf{7 6}$ & $\mathbf{0 . 5 9}$ & $\mathbf{0 . 8 9}$ & $\mathbf{0 . 9 7}$ & $\mathbf{0 . 8 9}$ \\
\hline $\mathbf{1}$ & 126 & 0.68 & 0.90 & 0.95 & 0.89 \\
\hline $\mathbf{1 , 5}$ & 185 & 0.61 & 0.85 & 0.95 & 0.87 \\
\hline $\mathbf{2}$ & 256 & 0.67 & 0.89 & 0.96 & 0.90 \\
\hline
\end{tabular}

Table 4. Bioinformatics imputation analysis, Affymetrix platform. Results of $H P$ alleles imputation from SNP haplotypes genotyped using an Affymetrix platform. Best prediction accuracy results are achieved in increments of 0,5 windows (in bold).

in a cohort of Italian subjects with ASD and healthy controls; ii) integrated PCR and microarray data of a subgroup of cases, to impute $H P$ alleles from flanking SNP haplotypes and discriminate the two $H P$ alleles.

Supposing that pre-HP2 corresponds to zonulin, we expected a very significant increase of HP2 allele frequency in subjects with ASD, or, at least, in cases suffering from GID. What we found is that HP2 allele prevailed in both subjects with ASD and controls. However, on the contrary to what we expected, there was a decrease in HP2 frequency when compared to the total number of controls $(\mathrm{P}<0.05)$ in the observed subjects with ASD. Interestingly, this was due to a relative increase of HP2 in the super-controls rather than a decrease of HP2 in subjects with ASD. Indeed, comparing HP allelic distribution among different Italian control cohorts we found super-controls show a significant increase of HP2: $\mathrm{P}<0.05$ when compared to NASD and $\mathrm{P}<0.005$ when compared to those of previous study of Bottini and collaborators and Napolioni and co-worker ${ }^{22,36}$. Consequently, the association between HP2 and ASD disappears when the super-controls are excluded from the data analysis and decreases when comparing the ASDs only with the super-controls (Table 2).

NASD controls were unrelated ASD negative while super-controls belong to a group of a "selected healthy population", resulting negative when screened for any mental condition (DSM-IV Axis I disorders). In the group of super-controls HP alleles frequency was significantly different from that of Italian and Caucasian healthy subjects $^{22,35,36}(\mathrm{P}=0.0020$ and 0.0006 , respectively), while allelic distribution of NASD controls was similar to that reported in literature. This suggests that super-controls are a niche within unrelated non-affected population, therefore it could not be representative for the NASD population. Furthermore, HP genotypes distribution within worldwide population differs upon ethnicity and geographical area ${ }^{42}$. For these reasons, $H P$ genotype data from previous studies on Italian population ${ }^{22,36}$ were included among NASD controls. No significant differences of allelic distribution were found between ASDs and NASD controls + neurotypical Italian populations. One limitation of this study is the sex-ratio imbalance between controls and subjects with ASD, with an equal distribution of males and females in controls and a prevalence of males in patients. Literature on sex distribution of HP allele within healthy population showed no significant difference in sex-based $H P$ allele distribution ${ }^{40,43}$. Indeed, the study of Zhao and colleagues declared no significant sex-based difference in the allelic distribution of $H P$; then again, the re-analysis of the data reported by Brackenridge showing that HP1-2 has different distribution between Australian males and females, revealed no significant differences in $H P$ allelic distribution by Fisher test $(\mathrm{P}=0.9066)$ (Supplementary Table S1). To better elucidate this important imbalance in our groups, we investigated the sex-based $H P$ allelic distribution within subjects with ASD and controls to solve this bias. No differences were found between $H P$ alleles in males and females in patients and total control, neither, separately, in NASD controls or in super controls. Taking together these findings, we conclude that $H P$ allelic distribution is not sex dependent and that in these investigations the $H P$ allele distribution is not related to sex imbalance between controls and patients with ASD.

A different age distribution between controls (adults) and patients (children) also exists. However, two previous study showed no significant association between age and $H P$ genotype in healthy subjects ${ }^{40,43}$.

Moreover, no allelic association was found between subjects with ASD and patients suffering from GID. This indicates that the $H P$ genotype does not represent a risk factor for ASDs pathogenesis, and that HP does not 


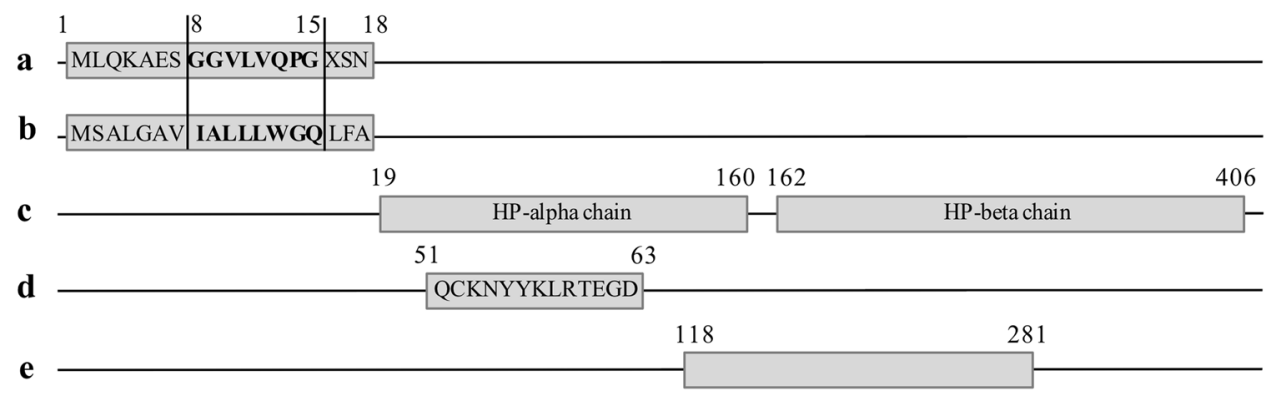

Figure 3. HP protein structure and keynote sequences. (a) Signal peptide according to Wang and colleagues ${ }^{13}$; (b) Signal peptide from UniProt accession number P00738; (c) Mature protein sequence from HP2 allele made up of alpha and beta chains; (d) Sigma-Aldrich declared immunogen sequence; (e) Sequence including capture antibody sequence of Human Zonulin ELISA Kit, Elabscience.

have a key role in the onset of GID in subjects with ASD. Furthermore, the significant decrease of HP1 allele in super-controls should be further investigated.

Our results do not reflect those of two previous studies that analyzed and found a relationship between zonulin and ASD. Indeed, Esnafoglu and collaborators ${ }^{29}$ observed a statistically significant increase of serum zonulin in a group of 32 subjects with ASD compared with 33 healthy controls, by ELISA (Elabscience). Then again, HP genotype was determined in a cohort of 46 subjects with ASD (20 of which with GIDs and 26 without GIDs) and 41 controls (6 of which with GIDs and 35 without GIDs) by plasma immunoblot. This study also found an association between HP2-2 and ASD with GIDs when compared with neurotypical developing children $(\mathrm{P}<0,01)$, and on the other hand found no association comparing ASD with GID with $\mathrm{ASD}^{30}$. However, these results were obtained from a very small cohort.

Considering the unexpected results of $H P$ allele frequency and ASD, we have made some considerations based on literature and the zonulin ELISA commercial kit. Zonulin is often and wrongly considered the alternative name of HP. Indeed, many authoritative databases, including UniProtKB [(P00738 (HPT_HUMAN)], Nextprot (NX_P00738) and NCBI (Gene ID 3234), report both HP and zonulin among "names", while only pre-HP2 belongs to the zonulin family. This has induced some authors to consider zonulin and HP as the same protein. Furthermore, the zonulin ELISA commercial kits and antibodies also reported indifferently HP or zonulin [i.e. Human Zonulin ELISA Kit, Elabscience, (Wuhan, Hubei Province, China) and IDK ${ }^{\circledR}$ Zonulin ELISA (Immundiagnostik AG, Germany)], and only after the publication of the study of Scheffler and colleagues, the datasheets were corrected reporting zonulin or $\mathrm{HP}$ as target protein.

Considering zonulin a family of proteins instead of a single on $\mathrm{e}^{27}$ modifies the interpretation of some previous literature. Indeed, zonulin quantification using commercial Human Zonulin ELISA kits was widely reported in different pathological conditions and the conclusions drawn on the basis of HP-zonulin identity. For instance, Esnafoglu and colleagues used the Human Zonulin ELISA Kit, Elabscience ${ }^{29}$ to quantify zonulin in the serum of subjects with ASD. The capture antibody of this kit recognizes a sequence within residues 118-281 of pre-HP2/ zonulin (uniprot ID: P00738) and the detection antibody within residues 104-346 (as referred by technical support, but not reported in the datasheet https://www.elabscience.com/PDF/Cate61/E-EL-H5560-Elabscience.pdf). These sequences may include both alpha (not specified if alpha-1 or alpha-2) and beta chain of HP, and is not specific for the signal peptide characterizing pre-HP2 (Fig. 3). Then again, the capture antibody of the IDK ${ }^{\circledR}$ Zonulin ELISA, recognizes a portion of zonulin previously reported by Wang and colleagues ${ }^{13}$, (datasheet http://www. immundiagnostik.com/fileadmin/pdf/Zonulin_K5601.pdf). Since this portion is not included in HP sequence, the antibody may recognize another zonulin family member, as suggested by Scheffler and colleagues ${ }^{27}$. Indeed, these authors, which integrated antibody capture experiments, mass spectrometry, and Western blot analysis, demonstrated that the IDK ${ }^{\circledR}$ Zonulin ELISA (based on a polyclonal antibody anti-zonulin) mainly recognize properdin, a potential second member of the zonulin family ${ }^{27}$. Interestingly, properdin (P27918 https://www. uniprot.org/uniprot/P27918) maps on Xp11.23 making male subjects more susceptible to its allelic or variant effect. Furthermore, this chromosome region includes three other genes related to autism: the calcium channel, voltage-dependent, alpha $1 \mathrm{~F}$ (CACNA1F) associated with syndromic autism and schizophrenia ${ }^{44}$, the phosphatase 1 , regulatory (inhibitor) subunit $3 \mathrm{~F}$ (PPP1R3F) from which rare mutations have been found in autism ${ }^{45}$ and histone deacetylase 6 (HDAC6) from which a partial skipping of exon 3 was found in a subject with $\mathrm{ASD}^{46}$.

Then again, gut dysbiosis has been described in subjects with $\mathrm{ASD}^{47-50}$ and gut microbial imbalance, that increases inflammation and microbial toxic metabolites production, is the strongest stimulus for the activation of the zonulin pathway with consequent intestinal permeability and trafficking of macromolecules through intestinal wall ${ }^{51}$. Moreover, gut microbes strongly participate in "microbiota-gut-brain-axis" (the microbiota-CNS cross-talk $)^{52}$ modulating inflammatory cytokines, neurotransmitters production and epigenetic factors such as RNA interference, DNA methylation and histone modification. So, microbiota alteration can produce negative effects on brain function via "gut-brain axis" dysregulation and, indeed, dysbiosis has been reported in many psychiatric conditions ${ }^{53}$ including ASD ${ }^{47-50}$. Recently, the role of mycotoxins has also been proposed in ASD pathogenesis $s^{54,55}$. Mycotoxins, worldwide contaminants of food with toxicological effects, induce intestinal permeability and inflammation and interact with gut microbiota resulting in impairment of gut health ${ }^{56}$. 
It is well known that the HP CNV is not in strong linkage disequilibrium with any individual SNP and therefore it is not successfully genotyped by SNP-array technologies ${ }^{57}$. This is likely due to the fact that $H P 1$ allele arose from recurrent deletions in HP2 allele ${ }^{58}$. In addition, HP1F and the left copy of HP2FS share a $300 \mathrm{bp}$ sequence identical to a segment of Haptoglobin-Related Protein (HPR) gene. This HPR sequence that contains many derived variants was probably transferred into $H P$ gene through a paralogous conversion event making $H P$ genotyping unpredictable by classic GWAS ${ }^{58}$. Thus, PCR or Real Time-PCR is generally performed for HP genotyping on very small cohorts.

Although HP1 allele arose many times, it has been shown that it is possible to impute HP alleles from SNP haplotypes with a high level of accuracy ${ }^{58}$. Indeed, alleles that are old and common today segregate on characteristic SNP haplotypes. In this paper, we assessed the performance of $H P$ alleles imputation from SNP haplotypes in our cohort. Overall, we observed a high accuracy in the prediction of HP genotypes in both autistic and healthy subjects screened by Illumina and Affymetrix platforms. In both groups, the prediction of HP2-2 genotype is more successful (accuracy is 0,97 in both platforms), on the other hand HP1-1 genotype is lower $(0,80$ and 0,59 , respectively).

Beagle prediction based on the Illumina microarray platform gives acceptable results and should be useful to genotype $H P$ for a large scale of subjects. Due to the lower accuracy of HP1-1 prediction, even if it represents a good accomplishment, a PCR genotyping to confirm HP1-1 prediction should be useful.

It is important to highlight that the intention of our study was to verify the distribution of HP2 allele in subjects with ASD and to relate this with GID comorbidity, also driven by the consideration of the increased levels of pre-HP2/zonulin in subject with ASD, measured by ELISA ${ }^{29}$. The unexpected results addressed the question on the possible dissimilarity between zonulin and HP2. During this investigation Scheffler and colleagues ${ }^{27}$ and Ajamian and colleagues ${ }^{59}$ preceded thus we confirm their statements.

\section{Conclusion}

Zonulin is a family of structurally and functionally associated proteins including pre-HP2 and properdin. On the contrary to our expectations, no correlation between $H P$ alleles and Italian ASD patients or between subjects with ASD and those patients suffering from GID was found. These results further support a recent study of Schleffer and colleagues ${ }^{27}$. They demonstrated that, within zonulin family members, properdin protein, rather than haptoglobin, is similar to Zot and possibly is involved in intestinal permeability. Interestingly, properdin maps on Xp11.23 show that male subjects are more prone to its effects. Further efforts should be dedicated to genotype and/or to sequencing this gene in subjects with ASD.

However, additional investigations with a wider number of cases and controls are necessarily required to confirm these results. For this purpose, the study proposes a bioinformatics method to predict $H P$ allele distribution starting from GWAS data.

Moreover, many issues concerning HP and zonulin definition and detection must still be thoroughly studied. In conjunction with new genetic and/or environmental and predisposing factors which may lead to or provoke leaky gut in autistic subjects.

Received: 11 September 2019; Accepted: 1 April 2020;

Published: 8 May 2020

\section{References}

1. Autism and Developmental Disabilities Monitoring Network Surveillance Year 2008 Principal Investigators; Centers for Disease Control and Prevention. Prevalence of autism spectrum disorders-Autism and Developmental Disabilities Monitoring Network, 14 sites, United States, 2008. MMWR Surveill Summ. 61, 1-19 (2012).

2. Smaga, I. et al. Oxidative stress as an etiological factor and a potential treatment target of psychiatric disorders. Part 2. Depression, anxiety, schizophrenia and autism. Pharmacol. Rep. 67, 569-80 (2015).

3. Rosenfeld, C. S. Microbiome Disturbances and Autism Spectrum Disorders. Drug Metab. Dispos. 43, 1557-71 (2015).

4. de Magistris, L. et al. Alterations of the intestinal barrier in patients with autism spectrum disorders and in their first-degree relatives. J. Pediatr. Gastroenterol. Nutr. 51, 418-24 (2010).

5. de Magistris, L. et al. Antibodies against food antigens in patients with autistic spectrum disorders. Biomed. Res. Int. 729349 (2013).

6. Berg, J. M. \& Geschwind, D. H. Autism genetics: searching for specificity and convergence. Genome Biol. 13, 247 (2012).

7. Adams, J. B., Johansen, L. J., Powell, L. D., Quig, D. \& Rubin, R. A. Gastrointestinal flora and gastrointestinal status in children with autism-comparisons to typical children and correlation with autism severity. BMC Gastroenterol. 16, 11-22 (2011).

8. Buie, T. et al. Recommendations for evaluation and treatment of common gastrointestinal problems in children with ASDs. Pediatrics. 125, S19-29(2010).

9. Hsiao, E. Y. Gastrointestinal issues in autism spectrum disorder. Harv. Rev. Psychiatry. 22, 104-11 (2014).

10. Akbari, P. et al. The intestinal barrier as an emerging target in the toxicological assessment of mycotoxins. Arch. Toxicol. 91, 1007-29 (2017).

11. Goldblum, S. E. et al. The active Zot domain (aa 288-293) increases ZO-1 and myosin 1C serine/threonine phosphorylation, alters interaction between ZO-1 and its binding partners, and induces tight junction disassembly through proteinase activated receptor 2 activation. FASEB J. 25, 144-58 (2011).

12. Tripathi, A. et al. Identification of human zonulin, a physiological modulator of tight junctions, as prehaptoglobin-2. Proc. Natl. Acad. Sci. USA 106, 16799-804 (2009).

13. Wang, W., Uzzau, S., Goldblum, S. E. \& Fasano, A. Human zonulin, a potential modulator of intestinal tight junctions. J. Cell Sci. 113, 4435-40 (2000)

14. Quaye, I. K. Haptoglobin, inflammation and disease. Trans. R. Soc. Trop. Med. Hyg. 102, 735-42 (2008).

15. Raynes, J. G., Eagling, S. \& McAdam, K. P. Acute-phase protein synthesis in human hepatoma cells: differential regulation of serum amyloid A (SAA) and haptoglobin by interleukin-1 and interleukin-6. Clin. Exp. Immunol. 83, 488-91 (1991).

16. Yang, F., Brune, J. L., Baldwin, W. D., Barnett, D. R. \& Bowman, B. H. Identification and characterization of human haptoglobin cDNA. Proc. Natl. Acad. Sci. USA 80, 5875-9 (1983).

17. Melamed-Frank, M. et al. Structure-function analysis of the antioxidant properties of haptoglobin. Blood. 98, 3693-8 (2001). 
18. Van Vlierberghe, H., Langlois, M. \& Delanghe, J. Haptoglobin polymorphisms and iron homeostasis in health and in disease. Clin. Chim. Acta. 345, 35-42 (2004).

19. Black, J. A. \& Dixon, G. H. Amino-acid sequence of alpha chains of human haptoglobins. Nature. 218, 736-41 (1968).

20. Buehler, P. W. et al. Haptoglobin preserves the CD163 hemoglobin scavenger pathway by shielding hemoglobin from peroxidative modification. Blood. 113, 2578-86 (2009).

21. Langlois, M. R. \& Delanghe, J. R. Biological and clinical significance of haptoglobin polymorphis in humans. Clin. Chem. 42, 1589-600 (1996).

22. Napolioni, V., Giannì, P., Carpi, F. M., Concetti, F. \& Lucarini, N. Haptoglobin (HP) polymorphisms and human longevity: a crosssectional association study in a Central Italy population. Clin. Chim. Acta. 412, 574-7 (2011).

23. Levy, A. P. et al. Haptoglobin: basic and clinical aspects. Antioxid. Redox Signal. 12, 293-304 (2010).

24. Pavón, E. J. et al. Proteomic analysis of plasma from patients with systemic lupus erythematosus: increased presence of haptoglobin alpha2 polypeptide chains over the alpha1 isoforms. Proteomics. 6, S282-92 (2006).

25. Dayan, L. et al. Haptoglobin genotype and endothelial function in diabetes mellitus: a pilot study. Eur. J. Appl. Physiol. 106, 639-44 (2009).

26. Fasano, A. Zonulin, regulation of tight junctions, and autoimmune diseases. Ann. N. Y. Acad. Sci. 1258, 25-33 (2012).

27. Scheffler, L. et al. Widely used commercial ELISA does not detect precursor of Haptoglobin2, but recognizes Properdin as a potential second member of the Zonulin family. Front. Endocrinol. (Lausanne). 9, 22 (2018).

28. Fasano, A. Intestinal permeability and its regulation by zonulin: diagnostic and therapeutic implications. Clin Gastroenterol Hepatol. 10, 1096-100 (2012).

29. Esnafoglu, E. et al. Increased serum Zonulin levels as an intestinal permeability marker in autistic subjects. J. Pediatr. 188, 240-44 (2017).

30. Rose, D. R. et al. Differential immune responses and microbiota profiles in children with autism spectrum disorders and co-morbid gastrointestinal symptoms. Brain Behav. Immun. 70, 354-68 (2018).

31. Lord, C. et al. ADOS-2-Autism Diagnostic Observation Schedule, 2nd ed.; Lord, C. \& Rutter, M., Eds.; Western Psychological Services: Torrance, CA, USA (2012).

32. Lord, C., Rutter, M. \& Le Couteur, A. Autism Diagnostic Interview-Revised: a revised version of a diagnostic interview for caregivers of individuals with possible pervasive developmental disorders. J. Autism Dev. Disord. 1994 24, 659-85 (1994).

33. Sheehan, D. V. et al. The Mini-International Neuropsychiatric Interview (M.I.N.I.): the development and validation of a structured diagnostic psychiatric interview for DSM-IV and ICD-10. J. Clin. Psychiatry. 59 Suppl 20, 22-33. quiz 34-57 (1998).

34. Folstein, M. F., Folstein, S. E. \& McHugh, P. R. Mini-mental state". A practical method for grading the cognitive state of patients for the clinician. J. Psychiatr. Res. 12, 189-98 (1975).

35. Koch, W. et al. Genotyping of the common haptoglobin Hp 1/2 polymorphism based on PCR. Clin. Chem. 48, 1377-82 (2002).

36. Bottini, N. et al. Haptoglobin genotype and natural fertility in humans. Fertil. Steril. 72, 293-6 (1999).

37. Sacchetti, E. et al. The GRM7 gene, early response to risperidone, and schizophrenia: a genome-wide association study and a confirmatory pharmacogenetic analysis. Pharmacogenomics J. 17, 146-54 (2017).

38. Browning, S. R. \& Browning, B. L. Rapid and accurate haplotype phasing and missing-data inference for whole-genome association studies by use of localized haplotype clustering. Am. J Hum. Genet. 81, 1084-97 (2007).

39. Browning, B. L. \& Browning, S. R. Genotype Imputation with Millions of Reference Samples. Am. J. Hum. Genet. 98, 116-26 (2016).

40. Brackenridge, C. J. A sex-dependent difference in the haptoglobin distributions of some Australian urban population samples, with a note on the method of Haldane for estimating the significance of the logarithm of a ratio of frequencies. J. Exp. Biol. Med. Sci. 49, 525-9 (1971).

41. Vanuytsel, T., Vermeire, S. \& Cleynen, I. The role of Haptoglobin and its related protein, Zonulin, in inflammatory bowel disease. Tissue Barriers. 1, e27321 (2013).

42. Carter, K. \& Worwood, M. Haptoglobin: a review of the major allele frequencies worldwide and their association with diseases. Int. J. Lab. Hematol. 29, 92-110 (2007).

43. Zhao, H., Zhang, G., Duan, Y. \& Yu, S. Haptoglobin types in Chinese ethnic groups. Hum. Hered. 43, 131-3 (1993).

44. Wei, J. \& Hemmings, G. P. A further study of a possible locus for schizophrenia on the X chromosome. Biochem. Biophys. Res. Commun. 344, 1241-5 (2006).

45. Piton, A. et al. Systematic resequencing of X-chromosome synaptic genes in autism spectrum disorder and schizophrenia. Mol. Psychiatry. 16, 867-80 (2011).

46. Piton, A. et al. Analysis of the effects of rare variants on splicing identifies alterations in GABAA receptor genes in autism spectrum disorder individuals. Eur. J. Hum. Genet. 21, 749-56 (2013).

47. Williams, B. L. et al. Impaired carbohydrate digestion and transport and mucosal dysbiosis in the intestines of children with autism and gastrointestinal disturbances. Plos One. 6, e24585 (2011).

48. Strati, F. et al. New evidences on the altered gut microbiota in autism spectrum disorders. Microbiome. 5, 24 (2017).

49. Campion, D., Ponzo, P., Alessandria, C., Saracco, G. M. \& Balzola, F. The role of microbiota in autism spectrum disorders. Minerva Gastroenterol. Dietol. 64, 333-50 (2018).

50. Pulikkan, J. et al. Gut microbial dysbiosis in Indian children with autism spectrum disorders. Microb. Ecol. 76, 1102-14 (2018).

51. Valitutti, F. \& Fasano, A. Breaking down barriers: how understanding celiac disease pathogenesis informed the development of novel treatments. Dig. Dis. Sci. 64, 1748-58 (2019).

52. Roman, P., Rueda-Ruzafa, L., Cardona, D. \& Cortes-Rodríguez, A. Gut-brain axis in the executive function of austism spectrum disorder. Behav. Pharmacol. 29, 654-63 (2018).

53. Alam, R., Abdolmaleky, H. M. \& Zhou, J. R. Microbiome, inflammation, epigenetic alterations, and mental diseases. Am. J. Med. Genet. B. Neuropsychiatr. Genet. 174, 651-60 (2017).

54. De Santis, B. et al. Study on the association among mycotoxins and other variables in children with autism. Toxins (Basel). 9, E203 (2017).

55. De Santis, B. et al. Role of mycotoxins in the pathobiology of autism: A first evidence. Nutr. Neurosci. 10, 1-13 (2017).

56. Liew, W. P. \& Mohd-Redzwan, S. Mycotoxin: its impact on gut health and microbiota. Front Cell Infect Microbiol. 8, 60 (2018)

57. Cahill, L. E. et al. Currently available versions of genome-wide association studies cannot be used to query the common haptoglobin copy number variant. J. Am. Coll. Cardiol. 62, 860-1 (2013).

58. Boettger, L. M. et al. Recurring exon deletions in the HP (haptoglobin) gene contribute to lower blood cholesterol levels. Nat Genet. 48, 359-66 (2016).

59. Ajamian, M., Steer, D., Rosella, G. \& Gibson, P. R. Serum zonulin as a marker of intestinal mucosal barrier function: may not be what it seems. Plos One. 14, e0210728 (2019).

\section{Acknowledgements}

Grants received in support of research work: Italian Ministry of Health (GR-2009-1570296); Italian Flagshipproject InterOmics (PB05); European Union's Horizon 2020 GEMMA project (grant agreement No 825033), www.gemma-project.eu. The Cell line and DNA bank of Rett Syndrome, X-linked mental retardation and other genetic diseases, member of the Telethon Network of Genetic Biobanks (project no. GTB18001), funded by 
Telethon Italy, and of the EuroBioBank network, provided us with specimens. We thank John Hatton of the Institute of Biomedical Technologies (CNR-ITB) for proofreading the manuscript. Two of several authors of this publication are members of the European Reference Network for rare malformation syndromes and rare intellectual and neurodevelopmental disorders, ERN-ITHACA.

\section{Author contributions}

F.A.C. participated in wet and statistical analysis, wrote the paper; E.M. bioinformatics analyses; M.G., C.M. generated SNP-array genotypes of the Brescia cohort; M.M. bioinformatics infrastructure development; M.L., N.G. wet analyses; A.B. patients recruitment; L.V. and M.M. ASD diagnosis; M.E.R. recruitment coordination; A.R. and F.C. provided DNA and diagnosis of Telethon patients; A. Mi. evaluation and recruitment of "super controls"; A.Ma. P.I. of the project funded by Italian Ministry of Health; L.M. responsible of the Italian Flagshipproject InterOmics (PB05), A.Me. conceived and designed the study, participated in wet and statistical analysis, wrote the paper. F.A. data interpretation and final revision. All the authors critically revised the manuscript.

\section{Competing interests}

The authors declare no competing interests.

\section{Additional information}

Supplementary information is available for this paper at https://doi.org/10.1038/s41598-020-64679-w.

Correspondence and requests for materials should be addressed to A.M.

Reprints and permissions information is available at www.nature.com/reprints.

Publisher's note Springer Nature remains neutral with regard to jurisdictional claims in published maps and institutional affiliations.

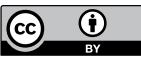

Open Access This article is licensed under a Creative Commons Attribution 4.0 International License, which permits use, sharing, adaptation, distribution and reproduction in any medium or format, as long as you give appropriate credit to the original author(s) and the source, provide a link to the Creative Commons license, and indicate if changes were made. The images or other third party material in this article are included in the article's Creative Commons license, unless indicated otherwise in a credit line to the material. If material is not included in the article's Creative Commons license and your intended use is not permitted by statutory regulation or exceeds the permitted use, you will need to obtain permission directly from the copyright holder. To view a copy of this license, visit http://creativecommons.org/licenses/by/4.0/.

(C) The Author(s) 2020, corrected publication 2023 